\title{
Effect of Climate on COVID-19 Incidence: A Cross-Sectional Study in Japan
}

\author{
Mikiro Kato ${ }^{1, *}$, Tomoko Sakihama ${ }^{2}$, Yoshio Kinjo ${ }^{3}$, David Itokazu ${ }^{4}$, Yasuharu Tokuda ${ }^{5}$ \\ 'Department of General Internal Medicine, Mito Kyodo General Hospital, University of Tsukuba, Tsukuba, Japan \\ ${ }^{2}$ International University of Health and Welfare Graduate School, Tokyo, Japan \\ ${ }^{3}$ Okinawa High School of Technology, Naha, Japan \\ ${ }^{4}$ Okinawa Asia Clinical Investigation Synergy, Naha, Japan \\ ${ }^{5}$ Muribushi Okinawa Center for Teaching Hospitals, Urasoe, Japan
}

Background: Effect of meteorological factors such as air temperature, humidity, and sunlight exposure on transmission dynamics of novel coronavirus disease 2019 (COVID-19) remains controversial. We investigated the association of these factors on COVID-19 incidence in Japan.

Methods: We analyzed data on reverse transcription polymerase chain reaction confirmed COVID-19 cases for each prefecture (total $=47$ ) in Japan and incidence rate was defined as the number of all reported cumulative cases from January 15 to March 17, 2020. Independent variables of each prefecture included three climatic variables (mean values of air temperature, relative humidity, and sunlight exposure), population elderly ratio, and the number of inbound travelers from China during February 2020. Multivariable-adjusted Poisson regression model was constructed to estimate COVID-19 incidence rate ratio (IRR) of independent variables.

Results: There was a total of 702 cases during the study period in Japan (population=125, 900,000). Mean \pm standard deviation values of meteorological variables were $7.12^{\circ} \mathrm{C} \pm 2.91^{\circ} \mathrm{C}$ for air temperature, $67.49 \% \pm 7.63 \%$ for relative humidity, and $46.77 \pm 12.55 \%$ for sunlight exposure. Poisson regression model adjusted for climate variables showed significant association between the incidence and three climatic variables: IRR for air temperature 0.854 (95\% confidence interval $[\mathrm{CI}], 0.804-0.907 ; \mathrm{P}<0.0001$ ), relative humidity 0.904 (95\% CI, 0.864-0.945; $\mathrm{P}<0.0001$ ), and sunlight exposure 0.973 (95\% CI, 0.951-0.997; $\mathrm{P}=0.026$ ).

Conclusion: Higher values of air temperature, relative humidity and sunlight exposure were associated with lower incidence of COVID-19. Public health interventions against COVID-19 epidemic in a country should be developed by considering these meteorological factors.

Keywords: COVID-19; SARS-Cov-2; Climate; Air Pollution; Meteorology

Received: December 2, 2020, Revised: January 13, 2021, Accepted: January 16, 2021

${ }^{*}$ Corresponding Author: Mikiro Kato https://orcid.org/0000-0003-4545-0745

Tel: +81-29-231-2371, Fax: +81-29-231-5137, E-mail: k.mikiro@md.tsukuba.ac.jp

'This article also accessed as preprints at https://doi.org/10.2139/ssrn.3612114. 


\section{INTRODUCTION}

Coronavirus disease (COVID-19) is currently spreading globally despite strong public health measures such as travel restrictions and lockdowns. Epidemics of viral respiratory tract infections, such as influenza, occur more frequently in winters, characterized by low air temperatures and dry air. ${ }^{1)}$ Climatic conditions, including temperature, humidity, and sunlight exposure, are considered to be important factors affecting the transmission of severe acute respiratory syndrome coronavirus 2 (SARS-CoV-2), and several studies have suggested a relationship between infection spread and winter climate. ${ }^{2-6)}$ In a study using global data, Wu et al. ${ }^{6)}$ noted that low air temperature and low relative humidity were associated with the number of daily new cases and COVID-19 mortality. However, regional patterns of COVID-19 outbreaks differ depending on the number of inbound travelers from a global epicenter, elderly population ratio, and population density. Thus, the results of these studies have not been consistent, and the impact of meteorological factors on viral spread is controversial.

Japan, located in the Northern Hemisphere, spreads across a wide range of longitudes. Thus, it is possible to examine the impact of climate on viral spread in Japan while considering the number of inbound travelers from a global epicenter, elderly population ratio, and population density. Therefore, in the current study, we aimed to investigate the impact of climate, specifically temperature, humidity, and sunlight exposure, on the spread of COVID-19 by using regional data from 47 prefectures during the initial phase (February and March 2020) of the COVID-19 outbreak in Japan.

\section{METHODS}

Data collection for each prefecture in Japan was conducted using published data from the public domains of the national government. As the dependent variable, the incidence of COVID-19 was defined as the cumulative number of reported cases in Japan, from the report of the first case in Japan on January 15, 2020 up to March 17, 2020. According to the Japanese case definition, clinically suspected patients with positive reverse transcription polymerase chain reaction results from respiratory specimens were considered cases. Our observation period ended in March 23, 2020 because public health measures, such as travel restrictions from China, were implemented at that time to mitigate the epidemic throughout the country. Cases involving the Diamond Princess cruise ship or charter flights from Wuhan, China, were excluded from the current study.

The independent variables in each prefecture included climate, particulate matter sized $\leq 2.5 \mu \mathrm{m}\left(\mathrm{PM}_{2.5}\right)$, elderly population ratio, and the number of inbound travelers based on government records in February 2020. We also included the population density of each prefecture in 2015. Data on the population density in 2020 were not available, as they have not yet been officially announced. Climate variables included mean air temperature $\left({ }^{\circ} \mathrm{C}\right)$, mean relative humidity $(\%)$, and mean relative sunshine duration (the ratio of actual to potential sunshine duration).

Based on the methods described by Ujiie et al., ${ }^{7)}$ the elderly population ratio (the ratio of the number of people aged $\geq 65$ years to the number of individuals aged 15-64 years) and the number of inbound travelers per 1,000 individuals in each prefecture were added as explanatory variables. Ujiie et al. ${ }^{7}$ allotted the number of travelers based on the people traveling from Tokyo International Airport (Chiba prefecture) to Chiba (the prefecture east to Tokyo prefecture), where the airport is located. However, in the current study, this number was allotted based on the number of people traveling to Tokyo, because most travelers were considered to travel directly to Tokyo from the airport through public transport (trains or buses) rather than through Chiba.

A multivariable-adjusted Poisson regression model was constructed to estimate the association between the dependent and independent variables. The incidence rate ratio (IRR) was determined. Two-tailed P-values less than 0.05 were considered statistically significant. STATA ver. 14.0 (Stata Corp., College Station, TX, USA) was used for all statistical analyses. The need for ethical approval was waived because all data belonged to the public domains of the Japanese government and personal data were not identified.

\section{RESULTS}

There were a total of 702 confirmed cases throughout Japan during the study period (population=125,900,000). The highest number of cases per 1 million people was identified in Hokkaido prefecture (26.7), followed by Aichi (15.8). No cases were reported in 13 prefectures. Figure

Table 1. Poisson regression model on the coronavirus disease 2019 incidence adjusted for climate variables, $\mathrm{PM}_{2.5}$ concentration, the number of inbound travelers from China, elderly population ratio, and population density

\begin{tabular}{lccc}
\hline \multicolumn{1}{c}{ Variable } & Incident rate ratio & 95\% confidence interval & P-value \\
\hline Air temperature & 0.873 & $0.823-0.926$ & $<0.0001$ \\
Humidity & 0.891 & $0.851-0.933$ & $<0.0001$ \\
Sunlight exposure & 0.973 & $0.950-0.997$ & 0.027 \\
PM $_{2.5}$ concentration & 1.032 & $0.960-1.109$ & 0.392 \\
Inbound traveler from China & 1.083 & $1.043-1.124$ & $<0.0001$ \\
Elderly population ratio & 0.993 & $0.954-1.034$ & 0.734 \\
Population density & 0.999 & $0.999-1.000$ & 0.001 \\
\hline
\end{tabular}

$\mathrm{PM}_{2.5}$, particulate matter sized $\leq 2.5 \mu \mathrm{m}$. 
1A shows the distribution of cases. The mean number of inbound travelers from China was 3.07 ( \pm standard deviation $[S D]=7.78$ ) in February 2020.

The largest number of inbound travelers was received in Osaka (37.09). There were 17 prefectures that received no inbound travelers. The highest elderly population ratio was observed in Akita (66.0), while the lowest was observed in Tokyo (34.3).

Mean \pm SD values of the climate variables in Japan in February 2020 were as follows: air temperature, $7.12^{\circ} \mathrm{C} \pm 2.91^{\circ} \mathrm{C}$; relative humidity, $67.49 \% \pm 7.63 \%$; and sunlight exposure, $46.77 \pm 12.55 \%$. The highest mean air temperature, relative humidity, and sunlight exposure were observed in Okinawa, Fukui, and Saitama and Gunma, respectively. The lowest mean air temperature, relative humidity, and sunlight exposure were observed in Hokkaido, Chiba, and Niigata, respectively.

Figure 1B-D show the distribution of climate data throughout Japan.
Table 1 shows the results of the Poisson regression model for COVID-19 incidence adjusted for climate variables, $\mathrm{PM}_{2.5}$ levels, number of inbound travelers from China, elderly population ratio, and population density. There was a significant association between COVID-19 incidence and climate variables, including air temperature, relative humidity, and sunlight exposure. Higher values of air temperature, relative humidity, and sunlight exposure were associated with a lower IRR for COVID-19: air temperature (IRR, 0.873; 95\% CI, 0.823-0.926; $\mathrm{P}<0.0001$ ); relative humidity (IRR, 0.891; 95\% CI, 0.851-0.933; $\mathrm{P}<0.0001$ ); and sunlight exposure (IRR, 0.973; 95\% CI, 0.950-0.997; $\mathrm{P}=0.027)$. A lower population density was associated with a higher IRR for COVID-19 (0.999; 95\% CI, 0.999-1.000; $\mathrm{P}=0.001)$.

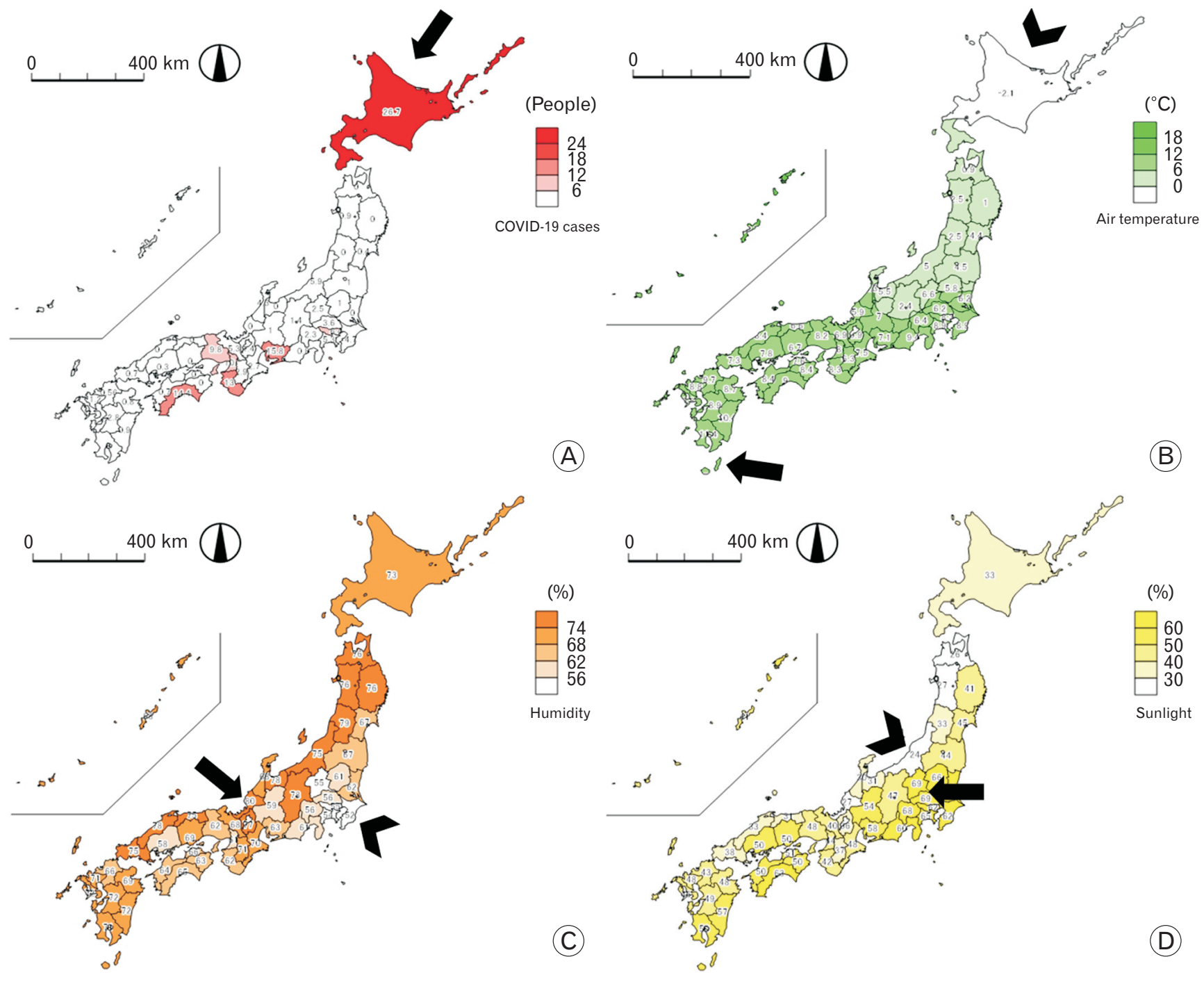

Figure 1. (A) Coronavirus disease 2019 (COVID-19) incidence rate per one million population during the study period. The highest case was identified in Hokkaido prefecture (black arrow). (B) Mean air temperature in February 2020. Highest mean air temperature was observed in Okinawa prefecture (black arrow), and lowest in Hokkaido (black arrowhead). (C) Mean relative humidity in February 2020. Highest relative humidity was observed in Fukui prefecture (black arrow), and lowest in Chiba (black arrowhead). (D) Mean sunlight exposure in February 2020. Highest mean sunlight exposure was observed in Saitama, Gunma prefecture (black arrow), and lowest in Niigata (black arrowhead). 


\section{DISCUSSION}

Based on a model of incidence data in Japan adjusted for $\mathrm{PM}_{2.5}$ levels, number of inbound travelers from China, elderly population ratio, and population density, our results suggest that higher values of air temperature, relative humidity, and sunlight exposure were associated with a lower IRR of COVID-19. Public health interventions, work-related activities for boosting the economy, could be planned by considering these meteorological factors during the ongoing COVID-19 pandemic. In a country like Japan, we may be able to relax lockdown restrictions and try to reintroduce certain economic activities in the summer. At the same time, we may need to be more alert and vigilant about viral transmission and to swiftly implement interventions during the winter. Our results confirmed the findings of recent studies on the effect of climate on the COVID-19 outbreak.

The primary route of transmission of SARS-CoV-2 involves respiratory droplets. Therefore, the transmission factor for SARS-CoV-2 could be similar to those of other viruses that are transmitted via respiratory droplets. ${ }^{8)}$ The survival of respiratory viruses is enhanced by decreases in air temperature. The relationship between high air temperature and virus inactivation has been observed for an enveloped virus in aerosols. ${ }^{9)}$ For human coronavirus 229E (alpha coronavirus) in aerosols, the survival rate was greater at low relative humidity than at high relative humidity. ${ }^{9)}$ Since SARS-CoV-2 is an enveloped virus and is categorized into the same family as other coronaviruses, these results are consistent with our results.

SARS-CoV-2 transmission via environmental contamination has also been documented. ${ }^{8)}$ Viruses do not replicate outside living cells, but the virus can persist on a contaminated environmental surface for several hours. Thus, the viability of the virus on a particular surface may be affected by climatic conditions, and there may be a relationship between viral transmission and different climatic conditions. A study on SARS-CoV, a beta-coronavirus that is genetically close to SARS-CoV-2, showed that high temperature and high relative humidity could synergistically inactivate SARS-CoV. Conversely, a low temperature and low relative humidity could prolong the viability of the virus on contaminated surfaces. ${ }^{10)}$

In the current study, sunlight exposure was associated with a lower incidence of COVID-19 in Japan. A study using global data by Gunthe et al. ${ }^{5)}$ reported that the ultraviolet index greatly lowered the spread and survival of the SARS-CoV-2. In SARS, ultraviolet irradiation, especially ultraviolet $C$ irradiation, efficiently eliminated infectivity. ${ }^{11,12)}$ The indirect interaction between sunlight exposure and severity of COVID-19 has also been shown. ${ }^{13)}$ It is noteworthy that all countries in the southern area below 35-degree north in the Northern Hemisphere have relatively low mortality rates. A potential association between an increased risk of severe COVID-19 and relatively low vitamin D levels due to low sunlight exposure has also been reported, indicating that vitamin D supplementation could prevent the cytokine storm and subsequent acute respiratory distress syndrome in patients with COVID-19. ${ }^{14)}$
Interestingly, our study showed an inverse relationship between population density and the IRR of COVID-19. Other studies that investigated the relationship between climate and COVID-19 transmissibility stated that other factors such as population density should also be taken into account, as the analysis of meteorological variables alone may lead to a misconception about COVID-19 transmissibility. ${ }^{15,16)}$ In contrast, Hamidi et al. showed that population density was not significantly associated with infection rates, possibly due to good adherence to social distancing guidelines. ${ }^{17)}$ Consistent with this finding, it has recently been reported that the incidence of COVID-19 is increasing in areas with lower population densities, such as rural areas. ${ }^{18)}$ Our results suggest the following hypotheses: although population density itself may contribute to the spread of COVID-19, it is necessary to consider other variables that have an inverse relationship with population density, such as adherence to wearing masks or social distancing, accessibility to health care systems, and poverty. ${ }^{17,19)}$

Our study has several limitations. First, our study involved ecological data, which may have introduced the issue of ecological fallacy. Second, our study was based on data from a single country, and the results may need validation in multiple countries. Third, we could not include other possible confounding factors, such as the volume of usage of air-conditioning in each prefecture. However, in a brief report on the COVID-19 outbreak on the Diamond Princess cruise ship, Xu et al. ${ }^{20)}$ suggested that air-conditioning played little role in the spread of COVID-19 on the ship and that most transmissions occurred through close contact in rooms without air-conditioning.

In conclusion, our study showed a possible association between a lower incidence rate of COVID-19 and specific climactic conditions, including higher values of air temperature, relative humidity, and sunlight exposure. The mechanisms underlying these associations may include reduced viral activity and transmission under such climatic conditions. To consolidate this finding, it is necessary to validate this relationship of COVID-19 in other countries. Healthcare workers, public health specialists, and policy makers may need to consider climate factors for developing a comprehensive plan for public health interventions against COVID-19 and increasing opportunities for economic activities.

\section{CONFLICT OF INTEREST}

No potential conflict of interest relevant to this article was reported.

\section{ORCID}

Mikiro Kato: https://orcid.org/0000-0003-4545-0745

Tomoko Sakihama: https://orcid.org/0000-0002-1160-1704

Yoshio Kinjo: https://orcid.org/0000-0002-6965-901X

Yasuharu Tokuda: https://orcid.org/0000-0002-9325-7934 


\section{REFERENCES}

1. Moriyama M, Hugentobler WJ, Iwasaki A. Seasonality of respiratory viral infections. Annu Rev Virol 2020;7:83-101.

2. Yao Y, Pan J, Liu Z, Meng X, Wang W, Kan H, et al. No association of COVID-19 transmission with temperature or UV radiation in Chinese cities. Eur Respir J 2020;55:2000517.

3. Qi H, Xiao S, Shi R, Ward MP, Chen Y, Tu W, et al. COVID-19 transmission in Mainland China is associated with temperature and humidity: a time-series analysis. Sci Total Environ 2020;728:138778.

4. Bashir MF, Ma B, Bilal, Komal B, Bashir MA, Tan D, et al. Correlation between climate indicators and COVID-19 pandemic in New York, USA. Sci Total Environ 2020;728:138835.

5. Gunthe SS, Swain B, Patra SS, Amte A. On the global trends and spread of the COVID-19 outbreak: preliminary assessment of the potential relation between location-specific temperature and UV index. Z Gesundh Wiss 2020 Apr 24 [Epub]. https://doi.org/10.1007/s10389-02001279-y.

6. Wu Y, Jing W, Liu J, Ma Q, Yuan J, Wang Y, et al. Effects of temperature and humidity on the daily new cases and new deaths of COVID-19 in 166 countries. Sci Total Environ 2020;729:139051.

7. Ujiie M, Tsuzuki S, Ohmagari N. Effect of temperature on the infectivity of COVID-19. Int J Infect Dis 2020;95:301-3.

8. Infectious Diseases Society of America. Infectious Diseases Society of America guidelines on infection prevention in patients with suspected or known COVID-19 [Internet]. Arlington (VA): Infectious Diseases Society of America; 2020 [cited 2020 Oct 28]. Available from: https:// www.idsociety.org/practice-guideline/covid-19-guideline-infectionprevention/.

9. Casanova LM, Jeon S, Rutala WA, Weber DJ, Sobsey MD. Effects of air temperature and relative humidity on coronavirus survival on surfaces. Appl Environ Microbiol 2010;76:2712-7.

10. Chan KH, Peiris JS, Lam SY, Poon LL, Yuen KY, Seto WH. The effects of temperature and relative humidity on the viability of the SARS corona- virus. Adv Virol 2011;2011:734690.

11. Duan SM, Zhao XS, Wen RF, Huang JJ, Pi GH, Zhang SX, et al. Stability of SARS coronavirus in human specimens and environment and its sensitivity to heating and UV irradiation. Biomed Environ Sci 2003;16:246-55.

12. Darnell ME, Subbarao K, Feinstone SM, Taylor DR. Inactivation of the coronavirus that induces severe acute respiratory syndrome, SARSCoV. J Virol Methods 2004;121:85-91.

13. Rhodes JM, Subramanian S, Laird E, Kenny RA. Editorial: low population mortality from COVID-19 in countries south of latitude 35 degrees North supports vitamin D as a factor determining severity. Aliment Pharmacol Ther 2020;51:1434-7.

14. Laird E, Kenny RA. Vitamin D deficiency in Ireland: implications for COVID-19: results from the Irish Longitudinal Study on Ageing [Internet]. Dublin: The Irish Longitudinal Study on Ageing; 2020 [cited 2020 Oct 28]. Available from: https://www.doi.org/10.38018/TildaRe.2020-05.

15. Jamshidi S, Baniasad M, Niyogi D. Global to USA county scale analysis of weather, urban density, mobility, homestay, and mask use on COVID-19. Int J Environ Res Public Health 2020;17:7847.

16. Mecenas P, Bastos RT, Vallinoto AC, Normando D. Effects of temperature and humidity on the spread of COVID-19: a systematic review. PLoS One 2020;15:e238339.

17. Hamidi S, Sabouri S, Ewing R. Does density aggravate the COVID-19 pandemic?: early findings and lessons for planners. J Am Plan Assoc 2020;86:495-509.

18. COVID-19 stats: COVID-19 incidence, by urban-rural classification: United States, January 22-October 31, 2020. MMWR Morb Mortal Wkly Rep 2020;69:1753.

19. Ranscombe P. Rural areas at risk during COVID-19 pandemic. Lancet Infect Dis 2020;20:545.

20. Xu P, Qian H, Miao T, Yen HL, Tan H, Kang M, et al. Transmission routes of COVID-19 virus in the Diamond Princess Cruise ship. medRxiv [Preprint] 2020 Apr 9 [Epub]. https://doi.org/10.1101/2020.04.09. 20059113 